\title{
Primary Cranial Vault Lymphoma Extending between Subcutaneous Tissue and Brain Parenchyma without Skull Destruction after Mild Head Trauma: A Case Report and Literature Review
}

\author{
Kengo Setta ${ }^{a}$ Takaaki Beppu $^{a}$ Yuichi Sato $^{a}$ Hiroaki Saura ${ }^{a}$ \\ Junichi Nomura ${ }^{a}$ Tamotsu Sugai $^{b}$ Kuniaki Ogasawara ${ }^{a}$ \\ ${ }^{a}$ Department of Neurosurgery, Iwate Medical University, Yahaba, Japan; ${ }^{b}$ Department of \\ Molecular Diagnostic Pathology, Iwate Medical University, Yahaba, Japan
}

Keywords

Primary cranial vault lymphoma $\cdot$ Diffuse large B-cell lymphoma $\cdot$ Head trauma

\begin{abstract}
Malignant lymphoma of the head rarely arises outside of the brain parenchyma as primary cranial vault lymphoma (PCVL). A case of PCVL that invaded from subcutaneous tissue into the brain, passing through the skull, and occurred after mild head trauma is reported along with a review of the literature. The patient was a 75-year-old man with decreased activity. One month before his visit to our hospital, he bruised the left frontal area of his head. Magnetic resonance imaging showed homogeneously enhanced tumors with contrast media in the subcutaneous tissue corresponding to the head impact area and the cerebral parenchyma, but no obvious abnormal findings in the skull. A biopsy with craniotomy was performed under general anesthesia. The pathological diagnosis was diffuse large B-cell lymphoma. On histological examination, tumor cells grew aggressively under the skin. Tumor cells invaded along the emissary vein into the external table without remarkable bone destruction and extended across the skull through the Haversian canals in the diploe. Tumor cells were found only at the perivascular areas in the dura mater and extended into the brain parenchyma. Considering the history of head trauma and the neuroimaging and histological findings, the PCVL in the present case arose primarily under the skin, passed though the skull and dura mater, and invaded along vessels and reached the brain.
\end{abstract}


Fig. 1. Bone window setting CT. CT, computed tomography.

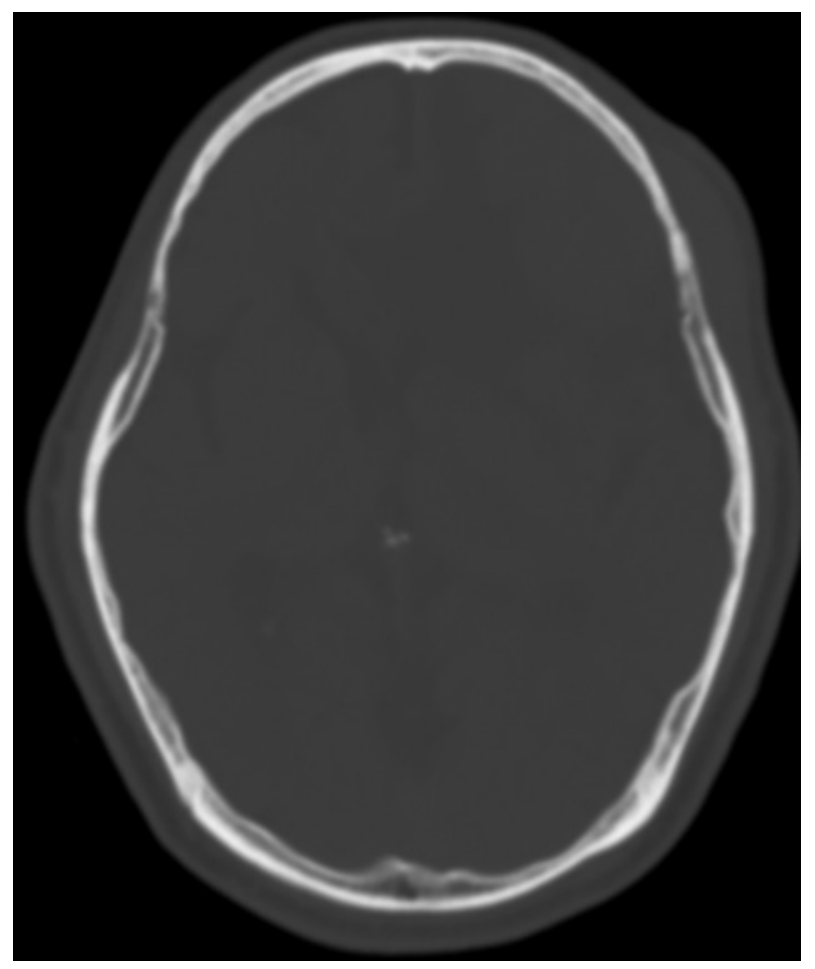

\section{Introduction}

Primary central nervous system lymphoma, accounting for approximately $3 \%$ of central nervous system tumors, generally occurs in the brain parenchyma, such as the periventricular white matter, corpus callosum, and basal ganglia. On the other hand, malignant lymphoma rarely arises in the cranial vault outside the brain parenchyma without being accompanied by systemic lymphoma. This pathological behavior has been defined as primary cranial vault lymphoma (PCVL) [1, 2]. PCVL extends from the subcutaneous tissue to the dura mater and may even invade into the brain parenchyma $[1,2]$. Furthermore, PCVL has been reported to arise extremely rarely in the site corresponding to past head trauma [3-6]. A rare case of PCVL that sandwiched apparently normal skull between subcutaneous and intracerebral lesions after mild head trauma is presented along with a review of the literature.

\section{Case Report}

The patient was a 75-year-old man with an otherwise unremarkable past history. One month before visiting our hospital, he slipped on the floor in the bathroom of his house and bruised the left frontal area of his head. Immediately after the injury, a lump with a blue bruise suggesting subcutaneous hemorrhage appeared on the injured area, but he paid no attention to it afterward. However, while the lump gradually enlarged, some symptoms, including gait disturbance, memory disturbance, and decreased activity, appeared and progressed rapidly, so his family members took him to our hospital. The subcutaneous lesion was an elastic hard, painless mass, but with normal colored skin. Blood examinations showed no immunodeficiency, virus infections, or other abnormalities. Computed tomography (CT) showed tumors with high density both in the subcutaneous and intracerebral regions of the left cranial vault, but no abnormalities in the skull bone (Fig. 1). On conventional magnetic resonance imaging, T2-weighted imaging demonstrated the intracerebral 

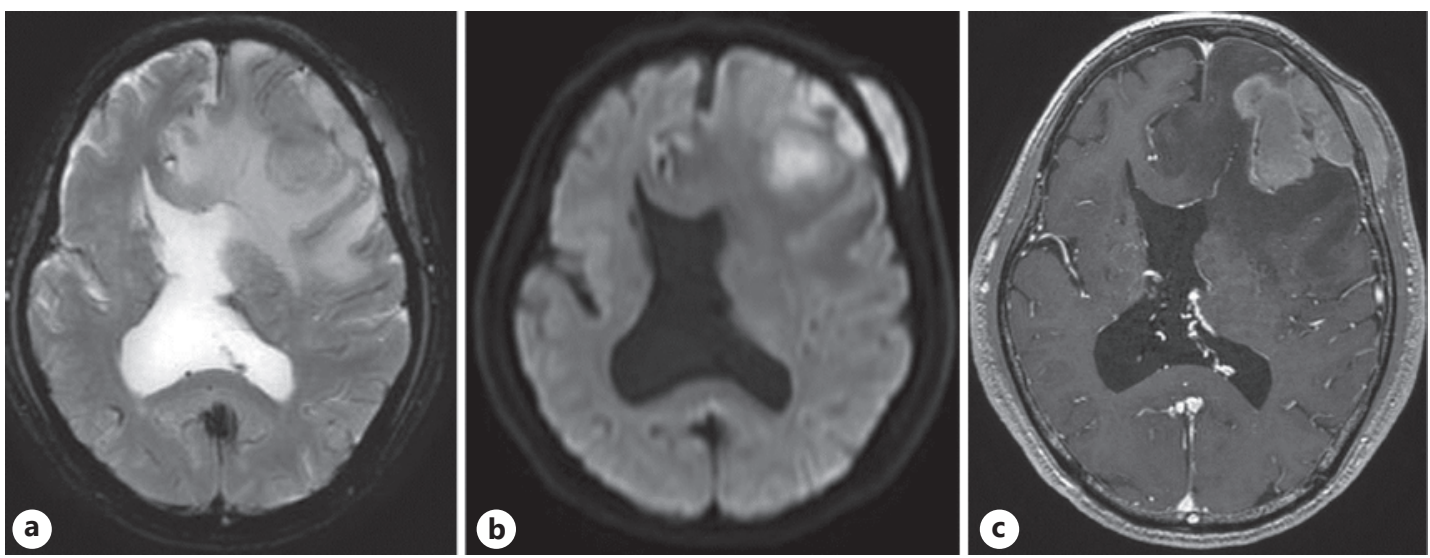

Fig. 2. Conventional MRI prior to surgery, T2WI (a), diffusion-weighted imaging (b), T1WI with contrast media (c). MRI, magnetic resonance imaging; T2WI, T2-weighted imaging; T1WI, T1-weighted imaging.

tumor with high signal intensity surrounded by widespread edema in the white matter, leading to a midline shift (Fig. 2a). The tumor showed strong high signal intensity on diffusion-weighted imaging (Fig. 2b). T1-weighted imaging with contrast media homogeneously enhanced both circumscribed subcutaneous and intracerebral tumors and showed a finding that mimicked the dural tail sign (Fig. 2c), but there were no obvious changes in the skull structure. Based on the imaging findings, the tumor was suspected to be a meningioma, metastatic brain tumor, or malignant lymphoma.

To make a histological diagnosis, a craniotomy was performed under general anesthesia. Intraoperatively, after a skin incision, the subcutaneous tumor was found to be growing in the temporal muscle layer. The skull seemed normal with a facade of usual tinge and hardness without evidence of erosion or destruction. The intracerebral tumor was easily separated from beneath white matter because the border between tumor and white matter was relatively clear. Since the intraoperative rapid pathological diagnosis was malignant lymphoma, removal of the tumor was stopped, and most of the intracerebral tumor was left to avoid injuring speech function. A consecutive block specimen comprising subcutaneous tumor, skull, dura mater, and intracerebral tumor was collected (Fig. 3a). On hematoxylin-eosin-stained preparations, the subcutaneous tumor consisted of neoplastic lymphocytes with poor cytoplasm, as well as large round nuclei, intermingling diffusely with temporal muscle fibers (Fig. 3b). Tumor cells invaded along the emissary vein into the external table of the skull and also filled in the perivascular spaces in the Haversian canals of the diploe (Fig. 3c). In the dura mater, tumor cells infiltrated sparsely, only around blood vessels (Fig. 3d). Tumor cells showed positive immunostaining for CD20 (Fig. 3e), CD79a, CD10, Bcl-2, Bcl-6, and MUM-1 in all segments of the tumor, and the pathological diagnosis was therefore diffuse large B-cell lymphoma. After the diagnosis, positron emission tomography with ${ }^{18} \mathrm{~F}$-fluorodeoxyglucose and bone marrow examination were carried out, but there were no abnormal findings in the body. The patient received chemotherapy with high-dose methotrexate for 3 cycles, followed by whole-brain and local boost radiotherapy at a total dose of $40 \mathrm{~Gy} / 20$ fractions, and the residual tumor disappeared completely. The patient has maintained relapse-free survival for 12 months after surgery.

\section{Discussion}

A literature search identified reports of a total of 63 cases of PCVL [1-5, 7-10]. In those 63 cases, PCVL arose predominantly in the parietal and frontal vaults of the head (24 parietal and 19 frontal) in middle-aged and elderly people. The male-to-female ratio showed a slight 


\section{Case Reports in Oncology}

\begin{tabular}{l|l}
\hline Case Rep Oncol 2021;14:1118-1123 \\
\hline DOI: 10.1159/000516272 & $\begin{array}{l}\text { @ 2021 The Author(s). Published by S. Karger AG, Basel } \\
\text { www.karger.com/cro }\end{array}$ \\
\hline
\end{tabular}

Setta et al.: Primary Cranial Vault Lymphoma after Mild Head Trauma

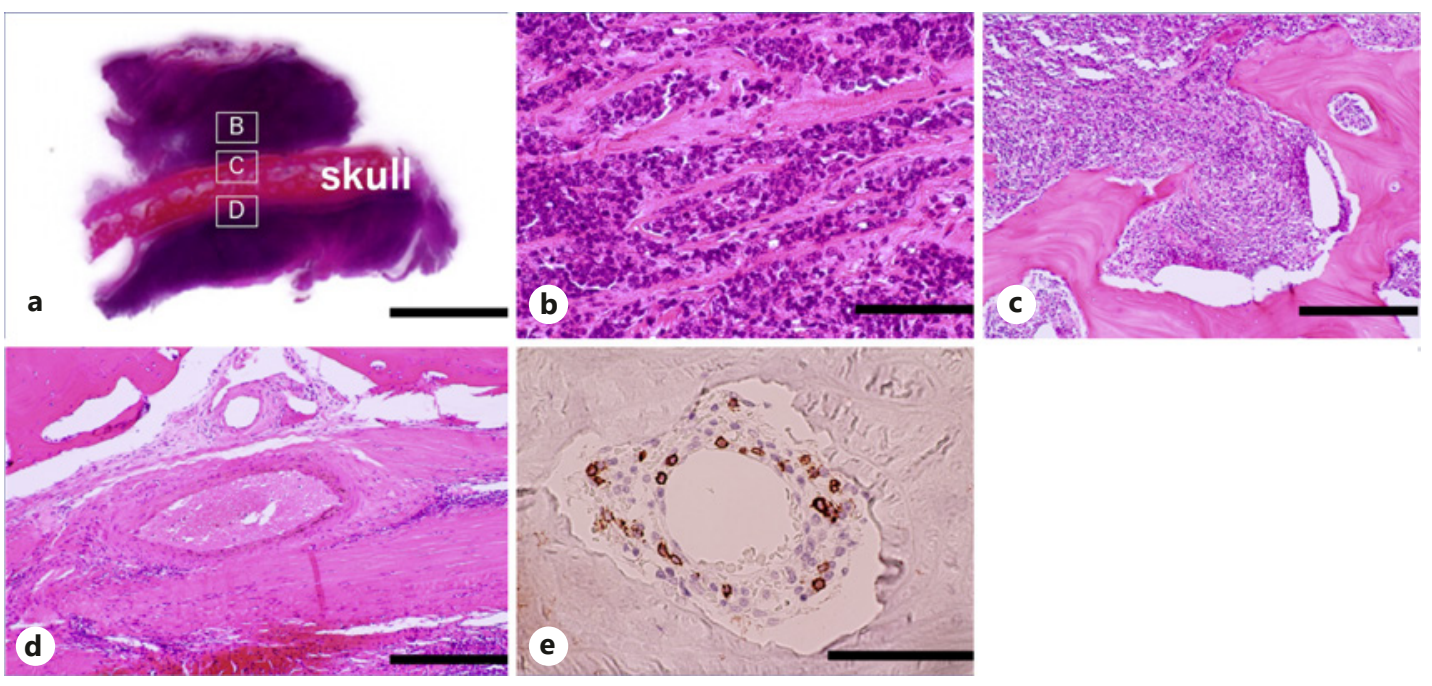

Fig. 3. a-d H\&E-stained preparations. a A consecutive block specimen between subcutaneous and intracerebral regions under low magnification. $\mathbf{b}$ Letters B, C, and D on Fig. $\mathbf{a}$ indicate the areas corresponding to the following. c Tumor cells intermingling with muscle fibers in the subcutaneous region. d Subcutaneous tissue-skull junction. Subcutaneous tumor cells invaded into a canal of the emissary vein in the external plate of the skull. Demonstration of the dura mater between the skull and subdural tumor. e The dura mater is seemingly intact, except for infiltration of tumor cells around vessels. CD20-positive tumor cells surrounding a vessel in a Haversian canal in the diploe. Scale bar, $10 \mathrm{~mm}$ in A, $250 \mu \mathrm{m}$ in B, $500 \mu \mathrm{m}$ in C, D, $100 \mu \mathrm{m}$ in E. H\&E, hematoxylin-eosin.

female predominance ( 24 men/39 women), and most cases were diffuse large B-cell lymphoma (41/63 cases) [1-9]. Thirteen of 63 cases $(20.6 \%)$ had PCVL invading the brain parenchyma [1-5, 7-9]. Since PCVL involving the dura mater and skull resembles meningioma and metastatic brain tumor, a diagnosis based on imaging findings is frequently difficult. Therefore, a pathological differential diagnosis of tissue specimens is essential [1]. Indeed, since the findings of conventional magnetic resonance imaging in the present case resembled those of meningioma and metastatic brain tumor, pathological diagnosis after a craniotomy was imperative.

PCVL must arise from subcutaneous tissue, skull, or dura mater. To determine which tissue is the primary site, however, may be difficult because PCVL extends across multiple layers between subcutaneous and subdural spaces on the initial imaging [7]. In the literature, PCVL originating from the dura mater was a low-grade marginal zone lymphoma in most cases, which frequently invades to the adjacent leptomeninges, but rarely to the scalp and skull [11]. Primary lymphoma of the skull shows a moth-eaten pattern, which is a slight change in the cortex of the skull in the early phase. Then, the skull is gradually eroded and completely destroyed in the late phase, while tumor invades the subcutaneous tissue [12]. In the pathological findings of the present case, the tumor cells grew aggressively in the temporal muscle, whereas the structures of the skull and dura mater were seemingly preserved, except for moderate infiltration of tumor cells along vessels. Since there was no significant destruction of the skull and dura mater, the possibility of PCVL arising from the skull or dura mater was doubtful in this case. In addition, the border between intracerebral tumor and beneath white matter was relatively clear during surgery. Therefore, the PCVL in the present case was likely to have originated primarily from the subcutaneous tissue.

Changes of the skull structure are not only seen in PCVL originating from the skull but also from the other tissue structures. In a report of CT findings in 39 cases of PCVL, 74\% of the cases showed osteolytic changes, and 5\% showed osteoclastic changes, whereas only $18 \%$ of the cases showed no bony changes [1]. In the literature, 11 of 13 cases whose PCVL 
invaded the brain parenchyma were documented to show osteolytic changes in the skull [1-5, 7-9]. PCVL in the present case showed rare invasive features without significant changes on imaging and histological findings in the skull, despite extending across the subcutaneous tissue to the brain parenchyma. Tashiro et al. [10] reported a case of PCVL extending across subcutaneous tissue and subdural space, despite no bony changes on CT, in which diffuse large B-cell lymphoma cells passed through the skull, invading along the emissary vein. Similarly, the PCVL cells in the present case probably originated in the subcutaneous tissue and passed through the skull, invading along veins in the diploe.

To the best of our knowledge, only 4 cases have been reported in the literature in which the PCVL was clearly described to occur at the vault corresponding to previous head trauma, such as the present case $[2,3,5,6]$. All 4 cases had suffered a mild head injury without skull fracture, and 2 of them noticed subcutaneous masses 1 month after injury, as in the present case. Bony changes were identified in all cases, but the present case. Three of 5 cases including the present case had PCVL that invaded the brain parenchyma. In the reports described above, the authors referred to 2 theories as to the mechanism for occurrence of lymphoma after head trauma. One is the tumorigenesis of lymphoma cells induced by chronic inflammation due to trauma $[5,6]$. The other is the presence of circulating lymphoma cells in the blood, which circulate in the vasculature all over the body without any development of solid lymphoma $[2,3,5,6]$. The former is a mechanism of tumorigenesis induced by chemical mediators, leading to genetic instability, dysmorphic cell reactions, and immune evasion during inflammatory repair processes over a long period $[13,14]$. The latter is a mechanism in which circulating lymphoma cells not eliminated immunologically are detained and proliferated in injured sites with impaired blood circulation and immunoresistance [15]. Considering that the PCVL in the present case developed a short time after trauma, the former is an unlikely mechanism in the present case. The latter mechanism of tumorigenesis of PCVL appeared to be applicable in the present case, if the PCVL in this case was caused by trauma.

\section{Statement of Ethics}

Written informed consent from the patient and the patient's family regarding the publication of this case was obtained.

\section{Conflict of Interest Statement}

The authors have no conflicts of interest to report.

\section{Funding Sources}

The authors have no funding sources in regard to this case report.

\section{Author Contributions}

Kengo Setta drafted the initial manuscript and submitted the final manuscript. Takaaki Beppu and Kuniaki Ogasawara revised the manuscript. Takaaki Beppu, Yuichi Sato, Hiroaki Saura, and Junichi Nomura performed medical examination, surgery, and treatment. Tamotsu Sugai made a pathological diagnosis.

\section{Karger'}




\section{References}

1 El Asri AC, Akhaddar A, Baallal H, Boulahroud O, Mandour C, Chahdi H, et al. Primary lymphoma of the cranial vault: case report and a systematic review of the literature. Acta Neurochir. 2012;154:257-65.

2 Tagawa M, Momita S, Irie J. Primary B cell lymphoma of the skull following head trauma; a case report. Rinsho Ketsueki. 1987;28:589-93.

3 Kumon Y, Sakaki S, Nakano K, Fukui K, Kohno H, Kurihara K. Primary malignant lymphoma of the skull presenting as a growing mass in the forehead; a case report. No Shinkei Geka. 1991;19:279-83.

4 Dai MS, Ho CL, Chen CY, Chen TM, Yu CP, Chao TY. Lymphoma of bone with initial presentation as a calvarial mass. Ann Hematol. 2000;79:700.

5 Huang J, Tang D, Xu Y, Wang X, Yu C, Dong Y. Head trauma complicated with primary cranial vault lymphoma: a case report. Medicine. 2019;98:e14465.

6 Morioka T, Tahima T, Nishio S, Nishie E, Fukui M, Okamura T. Malignant lymphoma of the scalp at the site of a previous blunt trauma: report of two cases. Surg Neurol. 1994;42:117-20.

7 Yadav BS, Bansal A, Sharma SC, Malhotra P, Ghosh N, Holdhoff M, et al . A 60-year-old Indian male with altered sensorium and extensive lymphoma of the scalp. Semin Oncol. 2013;40:e9-21.

8 Evliyaoglu C, Ilbay K, Ercin C, Ceylan S. Primary cranial vault lymphoma presenting as a traumatic subdural hematoma. Zentralbl Neurochir. 2006;67:26-9.

9 Salunke P, Garg R, Bal A, Kedia S, Bindal S. Primary malignant non-Hodgkin's lymphoma of the skull vault in an immunocompetent patient. Neurol India. 2013;61:201-4.

10 Tashiro R, Kanamori M, Suzuki H, Utsunomiya A, Meguro K, Uenohara H, et al . Diffuse large B cell lymphoma of the cranial vault: two case reports. Brain Tumor Pathol. 2015;32:275-80.

11 Iwamoto FM, Abrey LE. Primary dural lymphomas: a review. Neurosurg Focus. 2006;21:E5.

12 Xing Z, Huang H, Xiao Z, Yang X, Lin Y. CT, conventional, and functional MRI features of skull lymphoma: a series of eight cases in a single institution. Skeletal Radiol. 2019;48:897-905.

13 Küppers R. Mechanisms of B-cell lymphoma pathogenesis. Nat Rev Cancer. 2005;5:251-62.

14 Joyce JA, Fearon DT. T cell exclusion, immune privilege, and the tumor microenvironment. Science. 2015; 348(6230):74-80.

15 DeAngelis LM, Gutin PH, Leibel SA, Posner JB. Primary central nervous system lymphoma and other hemopoietic tumors. In: DeAngelis LM, Gutin PH, Leibel SA, Posner JB, editors. Intracranial tumors. Diagnosis and treatment. London: Martin and Dunitz Ltd; 2002. p. 323. 